\title{
Inferior Suprarenal Artery
}

National Cancer Institute

\section{Source}

National Cancer Institute. Inferior Suprarenal Artery. NCI Thesaurus. Code C52737.

A blood vessel arising from the renal artery that supplies blood to the suprarenal gland and ureter. 